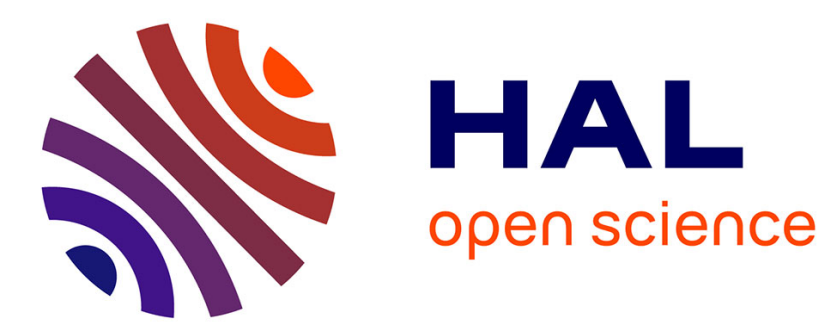

\title{
De-embedding multiplexers by Schur reduction
}

\author{
Sanda Lefteriu, Matteo Oldoni, Martine Olivi, Fabien Seyfert
}

\section{To cite this version:}

Sanda Lefteriu, Matteo Oldoni, Martine Olivi, Fabien Seyfert. De-embedding multiplexers by Schur reduction. CDC - Conférence on Decision and Control, Dec 2013, Florence, Italy. hal-00904794

\section{HAL Id: hal-00904794 \\ https://hal.inria.fr/hal-00904794}

Submitted on 15 Nov 2013

HAL is a multi-disciplinary open access archive for the deposit and dissemination of scientific research documents, whether they are published or not. The documents may come from teaching and research institutions in France or abroad, or from public or private research centers.
L'archive ouverte pluridisciplinaire HAL, est destinée au dépôt et à la diffusion de documents scientifiques de niveau recherche, publiés ou non, émanant des établissements d'enseignement et de recherche français ou étrangers, des laboratoires publics ou privés. 


\title{
De-embedding multiplexers by Schur reduction
}

\author{
Sanda Lefteriu ${ }^{1}$, Matteo Oldoni ${ }^{2}$, Martine Olivi $^{1}$ and Fabien Seyfert ${ }^{1}$
}

\begin{abstract}
This paper proposes a method to extract the characteristics of the filters composing a multiplexer. Scattering measurements of the multiplexer are given at some frequencies, together with information regarding the location of the transmission zeros of each filter. From these measurements we first compute a stable rational approximation of the scattering matrix (transfer function). Then we show that the chain matrices of the filters can be recovered, up to a constant matrix, by unchaining suitable components at each port of the multiplexer. The unchaining operation relies on a Schur reduction for an interpolation problem formulated for the structure thereby obtained, with the interpolation points being precisely the transmission zeros of the filter. Since transmission zeros are often located on the imaginary axis, we are dealing with a boundary interpolation problem.
\end{abstract}

\section{INTRODUCTION}

Diagnosis and tuning of microwave multiplexers in modern communication systems are becoming more and more challenging tasks in the development of telecommunication devices as their specifications tends to impose very high selectivity constraints. In this paper we present a method allowing to recover a rational representation of the filter's responses embedded in the multiplexer when starting from a set of scattering measurements of the overall multiplexing device. We consider a multiplexer composed of an $N+1$-port junction and $N$ filtering devices (Figure 1).

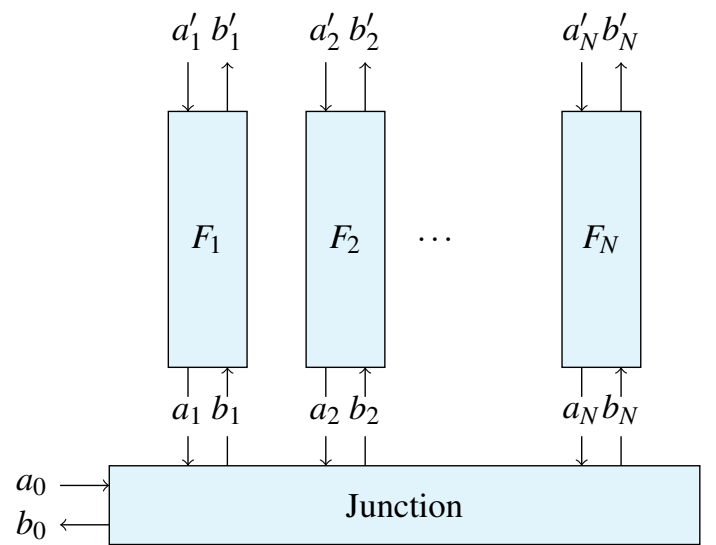

Fig. 1. Multiplexer

The term "filter" refers to a general 2-port device. Losslessness and reciprocity of the filters as well as the overall

\footnotetext{
${ }^{1}$ S. Lefteriu, M. Olivi and F. Seyfert are with INRIA, BP 93, 06902 Sophia-Antipolis Cedex, France Sanda.Lefteriu@inria.fr, Martine.Olivi@inria.fr, Fabien.Seyferteinria.fr

${ }^{2}$ M. Oldoni is with SIAE Microelettronica Spa, 21, via Buonarroti, 20093 Cologno Monzese, Italy Matteo.Oldoniesiaemic.com
}

multiplexer are assumed. These are usual assumptions in the design of microwave filters and multiplexers. It is moreover common practice to work in a rational framework. For more details on filters and multiplexers modeling, we refer the reader to [9]. Under these assumptions, the scattering matrices of these devices, which relate outgoing to incoming power waves, are (rational) lossless matrix functions. A lossless matrix function $G(s)$ is contractive on the right halfplane $\Pi^{+}$and $G(s)$ is unitary for $s$ on the imaginary axis,

$$
G(i w)^{*} G(i w)=I, \quad w \in \mathbb{R} .
$$

Note that $G(s)$ is then analytic in $\Pi^{+}$.

Throughout, if $M$ is a complex matrix, $M^{T}$ stands for its transpose and $M^{*}$ for its transpose conjugate; $M \geq 0$ (resp. $M>0$ ) means that $M$ is positive semidefinite (definite). If $F(s)$ is a matrix-valued function, then

$$
F^{*}(s)=F(-\bar{s})^{*}
$$

is the para-Hermitian conjugate of $F(s)$. Note that, on the imaginary axis, we have $F^{*}(i w)=F(i w)^{*}$. A lossless matrix function $G(s)$ is invertible and its inverse coincides with the para-Hermitian conjugate $G^{*}(s)$.

Linear fractional transformations (LFT) are very useful when describing the connections between networks. We first review some important facts concerning these transformations (see [3], [4] for details).

Let $\Theta(s)$ be a $2 p \times 2 p$ matrix-valued function partitioned as

$$
\Theta(s)=\left[\begin{array}{ll}
\Theta_{11}(s) & \Theta_{12}(s) \\
\Theta_{21}(s) & \Theta_{22}(s)
\end{array}\right]
$$

with blocks of size $p \times p$. The linear fractional transformation $T_{\Theta}$ associated with $\Theta(s)$ takes any $p \times p$ matrix $G(s)$ for which $\Theta_{21} G+\Theta_{22}$ is invertible to the $p \times p$ matrix given by

$$
T_{\Theta}(G)=\left(\Theta_{11} G+\Theta_{12}\right)\left(\Theta_{21} G+\Theta_{22}\right)^{-1} .
$$

If $G(s)$ is rational of McMillan degree $n$ and $\Theta(s)$ is rational of McMillan degree $m$, then $T_{\Theta} G(s)$ is rational of McMillan degree $\leq n+m$. The composition of mappings $T_{\Phi} \circ T_{\Psi}$ agrees with $T_{\Phi \Psi}$ on their joint domain of definition. If $F=T_{\Theta}(G)$, then $G=T_{\Theta(s)^{-1}}(F)$, which corresponds to

$$
T_{\Theta(s)^{-1}}(F)=\left(F \Theta_{21}-\Theta_{11}\right)^{-1}\left(\Theta_{12}-F \Theta_{22}\right) .
$$

We are mainly interested in linear fractional transformations associated with a $J$-lossless function $\Theta(s)$, since they preserve losslessness (see Theorem 1.1). We define Jlosslessness in the sequel. Let

$$
J=\left[\begin{array}{cc}
I_{p} & 0 \\
0 & -I_{p}
\end{array}\right] .
$$


A $2 p \times 2 p$ matrix-valued function $\Theta(s)$ is said to be (continuous-time) $J$-contractive if it satisfies

$$
J-\Theta(s)^{*} J \Theta(s) \geq 0 \text { for } s \in \Pi^{+},
$$

and $J$-lossless if, in addition, equality holds on the imaginary axis. We have the following result.

Theorem 1.1: If $G(s)$ is $p \times p$ lossless and the $2 p \times 2 p$ matrix $\Theta(s)$ is $J$-lossless, then $F=T_{\Theta(s)}(G)$ is $p \times p$ lossless.

The paper is organized as follows. Section II explains the relationship between the scattering matrix of the multiplexer $\sigma(s)$ and that of the junction by means of a linear fractional transformation involving the scattering matrices of the filters. This expression shows that, on one hand, the transmission zeros of a filter with positive real part are zeros of the non-diagonal entries of a column of $\Sigma(s)$, and on the other hand, the diagonal entry of this column evaluated at one of these transmission zeros is the same as the $(2,2)$ entry of that filter evaluated at that particular transmission zero. In section III and IV, we describe a method to extract the filters by unchaining elementary components at each port of the multiplexer. This unchaining operation amounts to perform a Schur reduction with interpolation condition at a transmission zero of the filter. Finally, in section V, we illustrate the method by the example of a diplexer $(N=3)$.

\section{CHAIN AND SCATTERING DESCRIPTION}

\section{A. Chain and scattering matrices of a filter}

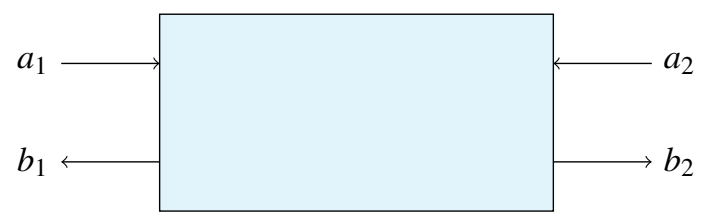

Fig. 2. Two-port device with incoming-outgoing waves

The scattering matrix $S(s)$ of a two port network (see Figure 2) relates outgoing waves to incoming waves.

$$
\left[\begin{array}{l}
b_{1} \\
b_{2}
\end{array}\right]=S\left[\begin{array}{l}
a_{1} \\
a_{2}
\end{array}\right]
$$

while the chain matrix $C(s)$ relates the signals at port 1 (left) to the signals at port 2 (right)

$$
\left[\begin{array}{l}
b_{1} \\
a_{1}
\end{array}\right]=C\left[\begin{array}{l}
a_{2} \\
b_{2}
\end{array}\right]
$$

so that cascading two networks amounts to multiplying their chain matrices. The chain and the scattering matrices are connected by a linear fractional transformation known as the Ginzburg transform (see [3], [6])

Proposition 2.1: The chain and the scattering matrices defined by (3) and (2) respectively are related by the LFTs

$C=T_{U}(S)$ with $U=\left[\begin{array}{ll|ll}1 & 0 & 0 & 0 \\ 0 & 0 & 1 & 0 \\ \hline 0 & 0 & 0 & 1 \\ 0 & 1 & 0 & 0\end{array}\right]=\left[\begin{array}{cc}U_{11} & U_{12} \\ U_{21} & U_{22}\end{array}\right]$.
This transformation is only defined when the $(2,1)$ entry $S_{21}(s)$ of $S(s)$ is non-zero. For a lossless matrix $S(s)$, the matrix $C(s)$ is $J$-lossless and its $(2,2)$ entry $C_{22}(s)$ has a stable numerator (roots with negative real part).

We assume that the filters are reciprocal, so that the scattering matrix is symmetric. We moreover assume that it satisfies $S(\infty)=I$, as it happens in most practical cases, though the unchaining algorithm described in the following sections holds for any value of $S(\infty)$. Under these hypotheses, the scattering matrix of the filters can be written as [2]

$$
S=\frac{1}{q}\left[\begin{array}{cc}
r & t \\
t & (-1)^{n} r^{*}
\end{array}\right]
$$

where $r(s)$ and $q(s)$ are monic polynomials of the same degree, say $n$, and $t(s)$ is a polynomial of degree at most $n-1$. The reciprocal polynomial of a polynomial $p(s)$ is defined as $p^{*}(s)=\overline{p(-\bar{s})}$ and $t(s)$ is auto-reciprocal: $t^{*}(s)=$ $(-1)^{n-1} t(s)$. Moreover, losslessness implies

$$
r r^{*}+t t^{*}=q q^{*}
$$

which gives the Feldtkeller equation $|r(i w)|^{2}+|t(i w)|^{2}=$ $|q(i w)|^{2}$ when restricted to the imaginary axis. The roots of $t(s)$ are the transmission zeros of the filter. They are located either on the imaginary axis, or as pairs of mirror image quantities in the complex plane. The associated chain matrix can be computed as

$$
C=\frac{1}{t}\left[\begin{array}{ll}
(-1)^{n+1} q^{*} & r \\
(-1)^{n+1} r^{*} & q
\end{array}\right]
$$

and reciprocity implies that its determinant is equal to 1 .

\section{B. The scattering matrix of the multiplexer}

Let the signals $a_{i}, b_{i}, a_{i}^{\prime}$ and $b_{i}^{\prime}$ be defined as in Figure 1. Let us denote by $\Sigma_{0}$ and $\Sigma$ the scattering matrices of the junction and the multiplexer respectively; The junction is assumed symmetrical, lossless and constant in frequency. We introduce the vectors

$$
a=\left[\begin{array}{c}
a_{0} \\
a_{1} \\
\vdots \\
a_{N}
\end{array}\right], b=\left[\begin{array}{c}
b_{0} \\
b_{1} \\
\vdots \\
b_{N}
\end{array}\right], a^{\prime}=\left[\begin{array}{c}
a_{0}^{\prime} \\
a_{1}^{\prime} \\
\vdots \\
a_{N}^{\prime}
\end{array}\right], b^{\prime}=\left[\begin{array}{c}
b_{0}^{\prime} \\
b_{1}^{\prime} \\
\vdots \\
b_{N}^{\prime}
\end{array}\right],
$$

so that

$$
\begin{aligned}
b & =\Sigma_{0} a \\
b^{\prime} & =\Sigma a^{\prime} .
\end{aligned}
$$

The scattering matrix $S^{(k)}(s)$ and the chain matrix $C^{(k)}(s)$ of filter number $k$ are defined as

$$
\left[\begin{array}{l}
a_{k} \\
b_{k}
\end{array}\right]=C^{(k)}\left[\begin{array}{l}
a_{k}^{\prime} \\
b_{k}^{\prime}
\end{array}\right], \quad\left[\begin{array}{l}
a_{k} \\
b_{k}^{\prime}
\end{array}\right]=S^{(k)}\left[\begin{array}{l}
b_{k} \\
a_{k}^{\prime}
\end{array}\right] .
$$

These matrix equalities can be rewritten in two dual chain and scattering relations

$$
\begin{aligned}
& {\left[\begin{array}{l}
a \\
b^{\prime}
\end{array}\right]=S\left[\begin{array}{l}
b \\
a^{\prime}
\end{array}\right]} \\
& {\left[\begin{array}{l}
a \\
b
\end{array}\right]=C\left[\begin{array}{l}
a^{\prime} \\
b^{\prime}
\end{array}\right]}
\end{aligned}
$$


where $S(s)$ and $C(s)$ are block-diagonal matrices given by

$$
S=\left[\begin{array}{cccc|cccc}
0 & 0 & \ldots & & 1 & 0 & \ldots & \\
0 & S_{11}^{(1)} & & & 0 & S_{12}^{(1)} & & \\
\vdots & & S_{11}^{(2)} & & \vdots & & S_{12}^{(2)} & \\
& & & \ddots & & & & \ddots \\
\hline 1 & 0 & \ldots & & 0 & 0 & \ldots & \\
0 & S_{21}^{(1)} & & & 0 & S_{22}^{(1)} & & \\
\vdots & & S_{21}^{(2)} & & \vdots & & S_{22}^{(2)} & \\
& & & \ddots & & & & \ddots
\end{array}\right]
$$

$$
C=\left[\begin{array}{cccc|cccc}
1 & 0 & \ldots & & 0 & 0 & \ldots & \\
0 & C_{11}^{(1)} & & & 0 & C_{12}^{(1)} & & \\
\vdots & & C_{11}^{(2)} & & \vdots & & C_{12}^{(2)} & \\
& & & \ddots & & & & \ddots \\
\hline 0 & 0 & \ldots & & 1 & 0 & \ldots & \\
0 & C_{21}^{(1)} & & & 0 & C_{22}^{(1)} & & \\
\vdots & & C_{21}^{(2)} & & \vdots & & C_{22}^{(2)} & \\
& & & \ddots & & & & \ddots
\end{array}\right]
$$

The matrix $S(s)$ is lossless while the matrix $C(s)$ is $J$-lossless where now $J$ is the signature matrix $\operatorname{diag}\left(I_{N+1},-I_{N+1}\right)$. Note that the filters and the junction being reciprocal implies that the matrix function $S(s)$ is in fact symmetric.

Eliminating $a$ and $b$ between (11) and (8), or alternatively between (10) and (8) we get

Proposition 2.2: The scattering matrix $\Sigma(s)$ of the multiplexer can be computed as a linear fractional transformation of the scattering matrix of the junction

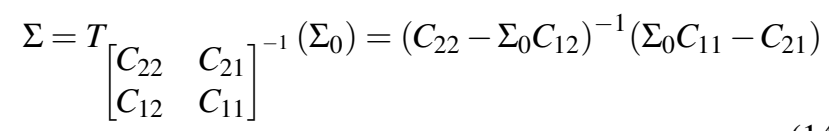

or alternatively as

$$
\Sigma=S_{22}+S_{21} \Sigma_{0}\left(I_{N}-S_{11} \Sigma_{0}\right)^{-1} S_{12}
$$

in which the $C_{i j}$ and $S_{i j}$ are the $(N+1) \times(N+1)$ blocks of the matrices $C(s)$ and $S(s)$ given by (13) and (12). These formulas were first studied in [12], [11].

Let $\sigma$ be a transmission zero of filter $k$ and let $e_{j}, j=$ $1, \ldots, N+1$ denote the $j$-th standard basis vector of $\mathbb{C}^{N+1}$. Thus,

$$
S_{12}^{(k)}(\sigma)=0 \text { and } S_{12}(\sigma) e_{k+1}=0 .
$$

Since $S_{22}(s)$ is diagonal, we have

$$
S_{22}(\sigma) e_{k+1}=S_{22}^{(k)}(\sigma) e_{k+1}
$$

Since

$$
\begin{aligned}
\Sigma(\sigma) e_{k+1} & =S_{22}(\sigma) e_{k+1}+S_{21} \Sigma_{0}\left(I_{N+1}-S_{11} \Sigma_{0}\right)^{-1} S_{12}(\sigma) e_{k+1} \\
& =S_{22}^{(k)}(\sigma) e_{k+1}+0=S_{22}^{(k)}(\sigma) e_{k+1} \text { by (16) and (17), }
\end{aligned}
$$

unless simplification occurs in (15) at the transmission zero $\sigma$. In fact, we show that simplification cannot occur for transmission zeros located in the closed right half-plane. Suppose that the matrix $I_{N+1}-S_{11}(\sigma) \Sigma_{0}$ is not invertible with vector $v \in \mathbb{C}^{N+1}$ in its nullspace. Therefore $\left(I_{N+1}-S_{11}(\sigma) \Sigma_{0}\right) v=0$, so $S_{11}(\sigma) \Sigma_{0} v=v$. Since the first row and column of $S_{11}$ are all zero, the vector $v$ should be of the form

$$
v=\left[\begin{array}{l}
0 \\
y
\end{array}\right], y \in \mathbb{C}^{N},
$$

where $y$ satisfies $\hat{S}_{11}(\sigma) \hat{\Sigma}_{0}(\sigma) y=y$ with

$$
\begin{aligned}
& \hat{S}_{11}=\left[\begin{array}{ccc}
S_{11}^{(1)} & & \\
& S_{11}^{(2)} & \\
& & \ddots
\end{array}\right], \text { and } \\
& \hat{\Sigma}_{0}=\Sigma_{0}(2: \text { end } 2: \text { end }) \text { in Matlab notation. }
\end{aligned}
$$

Since $\hat{\Sigma}_{0}$ is the submatrix of a unitary matrix, it is contractive. We shall assume that it is stictly contractive: for any non-zero vector $y,\left|\hat{\Sigma}_{0} y\right|<|y|$. It is a natural assumption in our context, it means that the junction actually feeds every filter of the device. The product $\hat{S}_{11}(\sigma) \hat{\Sigma}_{0}$ is also strictly contractive, as $\hat{S}_{11}$ is a diagonal matrix with entries smaller or equal to 1 in the closed right half-plane. This implies that for any vector $y$, we have the strict inequality $\left|\hat{S}_{11}(\sigma) \hat{\Sigma}_{0} y\right|<|y|$, so the equality $\hat{S}_{11}(\sigma) \hat{\Sigma}_{0}(\sigma) y=y$ cannot hold.

Under the assumption that $\hat{\Sigma}_{0}$ is strictly contractive, we have the following result:

Corollary 2.1: Every transmission zero $\sigma$ of filter $k$ with positive real part is a zero of the non-diagonal entries of the $(k+1)$ th column of $\Sigma(s)$. Moreover

$$
\left\{\begin{aligned}
\Sigma(\sigma) e_{k+1} & =S_{22}^{(k)}(\sigma) e_{k+1} \\
e_{k+1}^{T} \Sigma^{\prime}(\sigma) e_{k+1} & =\left[S_{22}^{(k)}\right]^{\prime}(\sigma)
\end{aligned}\right.
$$

By symmetry, $\sigma$ is also a zero of the non-diagonal entries of the $(k+1)$ th row of $\Sigma(s)$.

The property in Corollary 2.1 is a very important property which allows us to imagine interesting methods for the deembedding problem.

\section{UNCHAINING BY SCHUR REDUCTION}

This section shows that a Schur reduction at the transmission zeros offers a simple method to recover the filters. Transmission zeros of a filter usually belong to the imaginary axis, so that interpolation at the transmission zeros means boundary interpolation.

Thus, we first present a well-posed boundary interpolation problem and recall its solution (see [1], [10]).

Let $F(s)$ be a $p \times p$ rational lossless function, $\sigma \in i \mathbb{R}$ a point on the imaginary axis, and $u \in \mathbb{C}^{p}$ a given vector. Then the scalar expression

$$
\rho=-u^{*} F(\sigma)^{*} F^{\prime}(\sigma) u
$$

is nonnegative real: $\rho \geq 0$ and is known as the angular derivative of $F(s)$ at $\sigma$ in direction $u$. 
A well-posed boundary interpolation problem includes the value of the angular derivative $\rho$ and can be stated as follows. Find all the lossless functions $F(s)$ such that

$$
\left\{\begin{aligned}
F(\sigma) u & =v \\
v^{*} F^{\prime}(\sigma) u & =-\rho
\end{aligned}\right.
$$

where $\sigma$ belongs to the imaginary axis, $u$ and $v$ being unit vectors and $\rho$ a positive number (angular derivative). We have the following result:

Theorem 3.1: Any solution $F(s)$ of the (general angular derivative) interpolation problem (22) is given by

$$
F=T_{\Phi_{\sigma, u, v, \rho}}(\widetilde{F}),
$$

where $\Phi_{\sigma, u, v, \rho}$ is the $J$-lossless matrix function defined by

$$
\Phi_{\sigma, u, v, \rho}(s)=I_{2 p}-\frac{1}{\rho(s-\sigma)}\left[\begin{array}{c}
v \\
u
\end{array}\right]\left[\begin{array}{l}
v \\
u
\end{array}\right]^{*} J
$$

and $\widetilde{F}(s)$ is any $p \times p$ rational lossless function such that $\widetilde{F}(\sigma) u \neq v$. Moreover, the McMillan degree of $F(s)$ is equal to the McMillan degree of $\widetilde{F}(s)$ increased by 1 .

We now consider the particular case when $u$ and $v$ are collinear to a standard basis vector. The interpolation condition takes the form

$$
F(\sigma) e_{j}=\gamma e_{j}
$$

Note that, then,

$$
\gamma=F_{j j}(\sigma) \text { and } \rho=-\bar{\gamma} F_{j j}^{\prime}(\sigma)
$$

Due to the lossless assumption, moreover, $|\gamma|^{2}=1$. These are precisely the interpolation conditions we get when we compute the scattering matrix of the multiplexer at a transmission zero of one of the filters (see Corollary 2.1).

In this case the $J$-lossless matrix function (23) is given by

$$
\hat{\Theta}(s)=\left[\begin{array}{ccc|ccc}
I_{j-1} & 0 & 0 & 0 & 0 & 0 \\
0 & \Theta_{11} & 0 & 0 & \Theta_{12} & 0 \\
0 & 0 & I_{p-j} & 0 & 0 & 0 \\
\hline 0 & 0 & 0 & I_{j-1} & 0 & 0 \\
0 & \Theta_{21} & 0 & 0 & \Theta_{22} & 0 \\
0 & 0 & 0 & 0 & 0 & I_{p-j}
\end{array}\right]
$$

and can be viewed as an extension of the $2 \times 2$ matrix function

$$
\Theta(s)=I_{2}-\frac{1}{\rho(s-\sigma)}\left[\begin{array}{l}
\gamma \\
1
\end{array}\right]\left[\begin{array}{l}
\gamma \\
1
\end{array}\right]^{*} J .
$$

Then, Schur reduction only applies on $F_{j j}(s)$ while simplifying the common zero of the $j$ th column.

Proposition 3.1: Let $F(s)$ be a $p \times p$ lossless matrix of McMillan degree $n$, satisfying the boundary interpolation conditions

$$
\left\{\begin{aligned}
F(\sigma) e_{j} & =\gamma e_{j}, \\
e_{j}^{*} F^{\prime}(\sigma) e_{j} & =-\bar{\gamma} \rho .
\end{aligned}\right.
$$

The Schur reduction $\widetilde{F}=T_{\hat{\Theta}(s)^{-1}}(F)$ then produces a lossless matrix of degree $n-1$ such that $\widetilde{F}_{j j}=T_{\Theta(s)}{ }^{-1}\left(F_{j j}\right)$ and the set of zeros of $\widetilde{F}_{j l}(s)$, for $l \neq j$, is the set of zeros of $F_{j l}(s)$ minus $\sigma$.
Proof We may compute the linear fractional transformation $T_{\Lambda}(F)$ associated to $\Lambda(s)$, a degree one $J$-inner function of the general form ([3])

$$
\Lambda(s)=I_{2 p}+\lambda(s)\left[\begin{array}{l}
v \\
u
\end{array}\right]\left[\begin{array}{l}
v \\
u
\end{array}\right]^{*} J
$$

by the formula

$$
T_{\Lambda}(F)=F+\frac{\lambda(v-F u)\left(v^{*} F-u^{*}\right)}{1+\lambda\left(v^{*} F-u^{*}\right) u} .
$$

It is obtained by applying Formula A21 in [5] to compute the matrix inverse. In our case, if $c_{j}$ and $r_{j}$ denote the $j$ th column and the $j$ th row of $F(s), \widetilde{F}=T_{\hat{\Theta}(s)^{-1}}(F)$ is given by

$$
\widetilde{F}(s)=F(s)+\frac{\left(\gamma e_{j}-c_{j}(s)\right)\left(\bar{\gamma} r_{j}(s)-e_{j}^{T}\right)}{\rho(s-\sigma)+\bar{\gamma} F_{j j}(s)-1} .
$$

We thus have

$$
e_{j}^{T} \widetilde{F}(s) e_{j}=F_{j j}(s)+\frac{\left(\gamma-F_{j j}(s)\right)(\bar{\gamma}-1)}{\rho(s-\sigma)-\bar{\gamma} F_{j j}(s)-1},
$$

which is precisely equal to $T_{\Theta(s)}{ }^{-1}\left(F_{j j}\right)$. Moreover, for $l \neq j$

$$
e_{l}^{T} \widetilde{F}(s) e_{j}=\frac{\rho(s-\sigma) F_{l j}(s)}{\rho(s-\sigma)-\bar{\gamma} F_{j j}(s)-1},
$$

so that a double cancellation occurs at $\sigma$ due to the interpolation conditions. The same goes true for the $j$ th row.

Now, we shall see that if $F(s)=\Sigma(s)$ is the scattering matrix of a multiplexer, (20) asserts that $\Sigma(s)$ satisfies interpolation conditions of the form (25) for $j=k+1$ and $\sigma$ a transmission zero of filter $k$. Solving such an interpolation condition amounts to the factorization of the chain matrix of filter $k$ or, from a practical point of view, to unchaining an elementary component of this filter. Indeed, performing a Schur reduction associated with such an interpolation condition, we get a new scattering matrix $\widetilde{\Sigma}=T_{\hat{\Theta}(s)^{-1}}(\Sigma)$, and according to (14)

$$
\widetilde{\Sigma}=T\left(\left[\begin{array}{ll}
C_{22} & C_{21} \\
C_{12} & C_{11}
\end{array}\right] \hat{\Theta}\right)^{-1}\left(\Sigma_{0}\right) .
$$

The new chain matrix $\widetilde{C}(s)$ which relates the reduced scattering matrix $\widetilde{\Sigma}(s)$ to the scattering matrix $\Sigma_{0}$ of the junction is given by

$$
\widetilde{C}=C\left[\begin{array}{ll}
\hat{\Theta}_{22} & \hat{\Theta}_{21} \\
\hat{\Theta}_{12} & \hat{\Theta}_{22}
\end{array}\right] \text {. }
$$

In view of (13) and (24), $\widetilde{C}(s)$ is obtained from $C(s)$ by replacing the chain matrix $C^{(k)}(s)$ of filter $k$ by the matrix $\widetilde{C}^{(k)}(s)$ such that

$$
C^{(k)}=\widetilde{C}^{(k)}\left(I_{2}-\frac{1}{\rho(s-\sigma)}\left[\begin{array}{l}
1 \\
\gamma
\end{array}\right]\left[\begin{array}{l}
1 \\
\gamma
\end{array}\right]^{*} J\right) .
$$

The $J$-lossless matrix

$$
I_{2}-\frac{1}{\rho(s-\sigma)}\left[\begin{array}{l}
1 \\
\gamma
\end{array}\right]\left[\begin{array}{l}
1 \\
\gamma
\end{array}\right]^{*} J=\frac{1}{s-\sigma}\left[\begin{array}{cc}
s-\sigma-\frac{1}{\rho} & \frac{\bar{\gamma}}{\rho} \\
-\frac{\gamma}{\rho} & s-\sigma+\frac{1}{\rho}
\end{array}\right]
$$

is the chain matrix of the unchained elementary component. 
An equivalent first-order electrical circuit consisting of a pair of opposite phase shifters and a shunt arm (series of inductor and constant impedance or parallel of capacitor and constant admittance) can be easily derived from (28); this allows to sequentially build an electrical network representing the unchained blocks, which is often of practical interest in the modelling and tuning of microwave filtering devices.

We now handle the case of a zero at infinity. This case is of course essential for our study, since our filters satisfying $F(\infty)=I_{2}$ possess at least one transmission zero at infinity. An interpolation condition at infinity takes the form

$$
\left\{\begin{aligned}
F(\infty) u & =v \\
\lim _{s \rightarrow \infty} s^{2} u^{*} F(s)^{*} F^{\prime}(s) u & =\rho
\end{aligned}\right.
$$

Any solution $F(s)$ of the interpolation problem (29) is given by

$$
F=T_{\Phi_{\infty, u, v, \rho}}(\widetilde{F}),
$$

where $\Phi_{\infty, u, v, \rho}$ is the $J$-lossless matrix function defined by

$$
\Phi_{\infty, u, v, \rho}(s)=I_{2 p}-\frac{s}{\rho}\left[\begin{array}{l}
v \\
u
\end{array}\right]\left[\begin{array}{l}
v \\
u
\end{array}\right]^{*} J .
$$

and $\widetilde{F}(s)$ is a $p \times p$ rational lossless function of McMillan degree such that $\widetilde{F}(\infty) u \neq v$. Moreover, the McMillan degree of $F(s)$ is equal to the McMillan degree of $\widetilde{F}(s)$ increased by 1 . Note that, while the Schur recursion at a finite interpolation point preserves the value at infinity, this might not be the case for interpolation at infinity.

In the case $u=v=e_{j}$, the $J$-lossless function $\Phi_{\infty, e_{j}, \gamma e_{j}, \rho}(s)$ can again be viewed as an extension of the $J$-lossless function

$$
\Theta_{\infty}(s)=I_{2}-\frac{s}{\rho}\left[\begin{array}{l}
\gamma \\
1
\end{array}\right]\left[\begin{array}{l}
\gamma \\
1
\end{array}\right]^{*} J
$$

and Proposition 3.1 still holds true. Formula (26) becomes

$$
\widetilde{F}(s)=F(s)+\frac{s\left(\gamma e_{j}-c_{j}(s)\right)\left(\bar{\gamma} r_{j}(s)-e_{j}^{T}\right)}{\rho+s\left(\bar{\gamma} F_{j j}(s)-1\right)} .
$$

The chaining operation on filter $k$ becomes

$$
C^{(k)}=\widetilde{C}^{(k)}\left(I_{2}-\frac{s}{\rho}\left[\begin{array}{l}
1 \\
\gamma
\end{array}\right]\left[\begin{array}{l}
1 \\
\gamma
\end{array}\right]^{*} J\right) .
$$

The chain matrix of the unchained elementary component is then

$$
I_{2}-\frac{s}{\rho}\left[\begin{array}{l}
1 \\
\gamma
\end{array}\right]\left[\begin{array}{l}
1 \\
\gamma
\end{array}\right]^{*} J=\left[\begin{array}{cc}
1-\frac{s}{\rho} & \frac{\bar{\gamma}}{\rho} s \\
-\frac{\gamma}{\rho} s & 1+\frac{s}{\rho}
\end{array}\right] .
$$

Miror pairs of transmission zeros off the imaginary axis, if any, can be handled in a similar manner.

\section{A DE-EMBEDDING METHOD}

The idea is then to apply this unchaining operation of elementary components recursively to each filter.

Because of the block-diagonal form of the scattering matrix, if the transmission zeros are a-priori known, the unchaining operation described below can be performed at each port of the multiplexer independently. Since the degree drops at each Schur reduction, after a finite number of steps, having handled all the transmission zeros of each filter including those at infinity, we get a constant scattering matrix (of size $N+1 \times N+1$ ) that can be seen as possible model for the junction. We also get a rational representation of each filter which is unique up to a left multiplication by a constant chain matrix. The filter is given by its chain matrix in factored form (Potapov factorization). Each factor corresponds to one of the elementary (degree one) network that have been unchained.

\section{AN EXAMPLE: THE DIPLEXER}

We consider a diplexer with 2 filters, whose scattering matrices are given by (5), with polynomials

$$
\begin{gathered}
r^{(1)}(s)=(s-.1+.8 i)(s+.2+.85 i), t^{(1)}(s)=s-.5 i \text { and } \\
r^{(2)}(s)=(s+.3-.6 i)(s-.2+.65 i), t^{(2)}(s)=s-.1 i,
\end{gathered}
$$

respectively. Through a spectral factorization and using that $q^{(1)}$ and $q^{(2)}$ are stable, we obtain that

$$
\begin{gathered}
q^{(1)}(s)=(s+1.4023+1.5595 i)(s+0.4017+0.0905 i) \text { and } \\
q^{(2)}(s)=(s+0.6701+0.42 i)(s+0.4602-0.37 i) .
\end{gathered}
$$

Due to the low-pass model of a filter that we use here, the scattering matrices have complex coefficients. The junction is chosen constant:

$$
\Sigma_{0}=\left[\begin{array}{rrr}
-\frac{1}{3} & \frac{2}{3} & \frac{2}{3} \\
\frac{2}{3} & -\frac{1}{3} & \frac{2}{3} \\
\frac{2}{3} & \frac{2}{3} & -\frac{1}{3}
\end{array}\right],
$$

which corresponds to an ideal connection of all the filters' ports to the common port. We perform $N_{\text {meas }}=100$ linearly distributed measurements on the imaginary axis in the interval $[0, i]$ to obtain the scattering matrix $\Sigma(s)$ of the diplexer, using (14).

Since this is an academic example, we start from a-priori known filters and compute the scattering measurements of the diplexer. In a real-world setting, S-parameter measurements are given, while the filters are to be found. The problem amounts to determining the two filters from the knowledge of:

- the S-parameter measurements of the diplexer

- the transmission zeros $\sigma^{(1)}=.5 i$ and $\sigma^{(2)}=.1 i$, respectively, as well as the fact that both filters have a transmission zero at infinity.

From the measurements of $\Sigma(s)$, we build a rational approximation using the Loewner framework [7]. In this framework, the set of measurements are split into two disjoint subsets, which are used to build the Loewner and shifted Loewner matrices. The singular value drop of these matrices reveals the order of the underlying structure (see Fig. 3). We deduce that there is a strictly rational system of order 4 , while the singular value drop of the shifted Loewner matrix indicates a polynomial part of rank 3 , as expected.

We proceed by unchaining the transmission zeros of the first filter. Using the rational model for $\Sigma(s)$, we compute $\gamma$ and $\rho$ in (25) associated to $\sigma^{(1)}$, as well as the inverse of the elementary factor in (28). Next, we recompute the scattering 


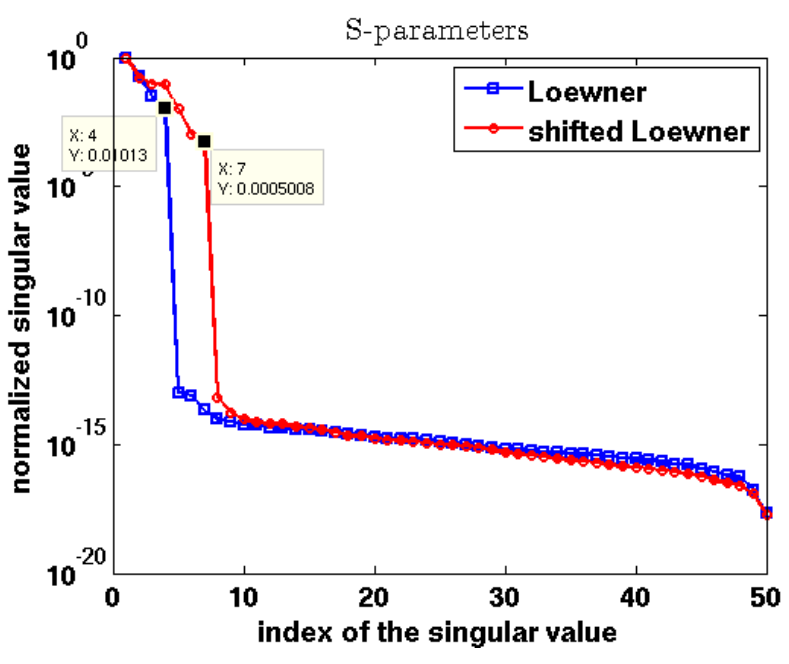

Fig. 3. Drop of the singular values of the Loewner and shifted Loewner matrices [7] built from the S-parameters of the diplexer

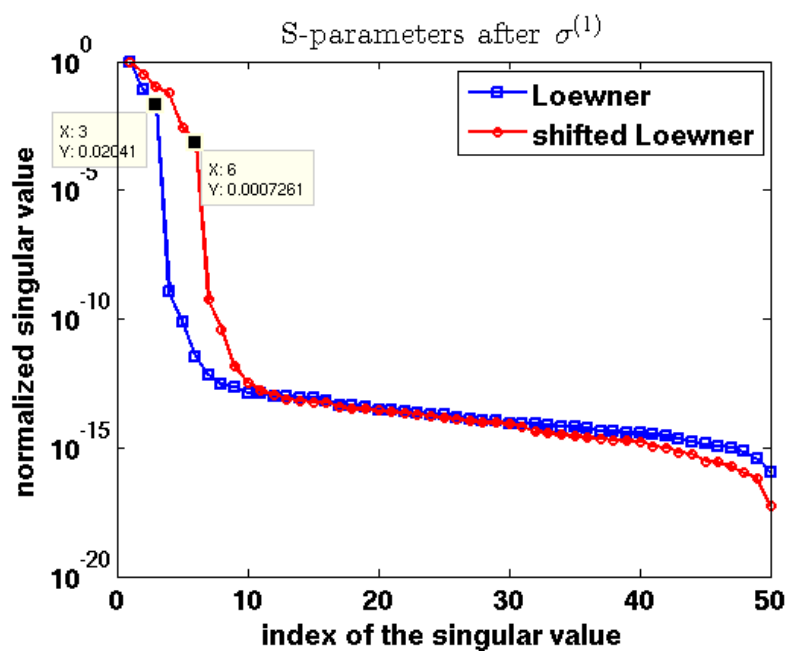

Fig. 4. Drop of the singular values of the Loewner and shifted Loewner matrices [7] built from the S-parameters of the diplexer after extracting $\sigma^{(1)}$

coefficients using (26) to obtain the updated quantities after $\sigma^{(1)}$ has been unchained. We use the new measurements to compute the Loewner matrix pencil whose singular value drop (see Fig. 4) shows a drop in degree by 1 . The new model allows us to unchain the infinite zero following the same steps as for $\sigma^{(1)}$. After these two unchaining steps, by computing the product of the elementary chain matrices, we know filter 1 up to a constant J-lossless matrix $J^{(1)}$.

The current S-parameters show a drop of two degrees with respect to the original quantities. We use these parameters to build a rational model which will be used to compute $\gamma$ and $\rho$ in (25) associated to the finite transmission zero of filter 2, namely $\sigma^{(2)}$. The same steps are performed as in the previous case, and we are able to determine the second filter as a product of elementary chain matrices, up to a constant J-lossless matrix $J^{(2)}$. After extracting all transmission zeros for both filters, the leftover S-parameters are constant.

\section{CONCLUSION}

In this paper, we have presented an original method to handle the de-embedding of filters in a multiplexer when starting from S-parameters measurements and the location of the transmission zeros of each filter. Unlike other approaches [12], [11] the latter does not assume the knowledge of the scattering matrix of the junction. This might render this methodology particularly attractive in practice, where the junction's S-parameters are hardly available. The method has been validated on a simple theoretical example, in a perfectly lossless framework for the filters and the junction: this is necessary for the Schur reduction to work. Future efforts will focus on manners to handle lossy devices, by replacing for example Schur interpolation by rational interpolation. A procedure to determine the transmission zeros of each filter is also under study.

\section{REFERENCES}

[1] J.A. Ball, I. Gohberg, and L. Rodman. Interpolation of rational matrix functions, volume 45 of Operator Theory: Advances and Applications. Birkhäuser, 1990.

[2] S. Bila, R.J. Cameron, P. Lenoir, V. Lunot, and F. Seyfert. Chebyshev synthesis for multi-band microwave filters. In 2006 IEEE MTT-S International Microwave Symposium Digest, pages 1221-1224, 2006.

[3] H. Dym. J-contractive matrix functions, reproducing kernel spaces and interpolation, volume 71 of CBMS lecture notes. American Mathematical Society, Rhode Island, 1989.

[4] B. Hanzon, M. Olivi, and R.L.M. Peeters. Balanced realizations of discrete-time stable all-pass systems and the tangential Schur algorithm. Linear Algebra and its Applications, 2006.

[5] T. Kailath Linear Systems. Prentice-Hall, 1980.

[6] H. Kimura. Chain-Scattering Approach to $\mathrm{H}^{\infty}$-Control. Birkhäuser, 1997.

[7] S. Lefteriu and A.C. Antoulas. A new approach to modeling multiport systems from frequency-domain data. Computer-Aided Design of Integrated Circuits and Systems, IEEE Transactions on, 29(1):14-27, Jan.

[8] Vincent Lunot. Techniques d'approximation rationnelle en synthèse fréquentielle : problème de Zolotarov et algorithme de Schur. $\mathrm{PhD}$ Thesis, Université de Provence, 2008.

[9] Matteo Oldoni. Synthesis and modeling techniques for microwave filters and multiplexers. PhD Thesis, Politecnico di Milano, 2012.

[10] Ralf L.M. Peeters, Martine Olivi, and Bernard Hanzon. Continuoustime lossless systems, boundary interpolation and pivot structures. In SSSC- 5th Symposium on System Structure and Control - 2013, Grenoble, France, February 2013.

[11] M. Oldoni, F. Seyfert, G. Macchiarella, and D. Pacaud. De-embedding response of filters from diplexer measurements. International Journal of RF and Microwave Computer-Aided Engineering, 2012.

[12] M. Oldoni, F. Seyfert, G. Macchiarella, and D. Pacaud. Deembedding of filters' responses from diplexer measurements Microwave Symposium Digest (MTT), 2011 IEEE MTT-S International, 2011 